\title{
Impatiens cyclosepala Hook. f. ex W.W. Sm. - a new species record for the flora of India from Arunachal Pradesh
}

\section{Umeshkumar L. Tiwari*, Krishna Chowlu \& Souravjyoti Borah}

Botanical Survey of India, Arunachal Pradesh Regional Centre, Senki View, Itanagar, India

* corresponding author (e-mail: tigerumesh11@gmail.com)

\begin{abstract}
The paper reports Impatiens cyclosepala Hook. f. ex W.W. Sm. as a new addition for India from Arunachal Pradesh. The species was, till to-date, known only from more than a 100 years old collection from NW Yunnan.
\end{abstract}

Key words: new record, rediscovery, India, Impatiens cyclosepala

The Himalayas have been reported with ca. 10000 plant species, among these approximately $31 \%$ are endemic to the region. Due to inaccessibility and difficult terrain of north-eastern region of India, floristically, these plants are not well documented. In India, genus Impatiens is represented by more than 210 species (Vivekananthan et al. 1997; Bhaskar 2012) of which 137 species are endemic (Vivekananthan et al. 1997; Swaminathan et al. 2001) and have not been well documented from Arunachal Pradesh, a part of the Eastern Himalaya. In recent years, many new species of Impatiens were discovered from the region (Gogoi \& Borah 2013a, 2014, 2015a, 2015b, 2015c, 2015d, 2015e, 2017a, 2017b, Gogoi et al. 2017a, 2017b; Hareesh et al. 2016a, 2016b, 2017a, 2017b; Hareesh \& Sabu 2017; Lidén \& Bharali 2017; Hareesh et al. 2017), as well as rediscoveries (Gogoi et al. 2013), and distribution extensions (Gogoi \& Borah 2013b, Borah et al. 2015, Gogoi et al. 2015a, 2015b, 2016) were reported.

During two floristic expeditions to East and West Kameng districts of Arunachal Pradesh in 2016 and 2017, respectively, one species of Impatiens with a yellowish lower sepal, pink to purple petals and the upper petal with two horn-like appendages was collected. After critical examination of specimens and search in literature dealing with Impatiens of the Indian subcontinent and adjacent areas and including the paper mentioned above and Hooker $(1875,1908 \mathrm{a}, 1908 \mathrm{~b}, 1910)$, Toppin (1920), Grey-Wilson (1989, 1991), Vivekananthan et al. (1997), Swaminathan et al. (2001), Huang et al. (2003), Huang (2006), Chen et al. (2008), Pusalkar \& Singh (2010), Dessai \& Janarthanam (2011), Bhaskar (2012), $\mathrm{Yu}$ (2012), Ruchisansakun et al. (2014), it turned out that this was Impatiens cyclosepala Hook. f. ex W.W. $\mathrm{Sm}$. and that the species has not been reported from India so far. Hence, it is reported here with descriptions and colour illustrations so as to enable field botanists, foresters, environmentalists, herbalists, etc. to identify them in the field.

Impatiens cyclosepala Hook. f. ex W.W. Sm., Notes Roy. Bot. Gard. Edinburgh 8: 338. 1915; Chen et al., Fl. China, 12: 53. 2007. Fig. $1 \& 2$.

H o l o type: China, West Yunnan, 8-9000 ft, 1906, G. Forrest 4271 E (E00313641), image!

Plant annual erect, $60-70 \mathrm{~cm}$ tall, stem greenish brown, puberulent, white hairs, turgid at nodes, laxly branched. Leaves alternate, petiolate, petiole 1-6 cm long; leaf blade 3-14×3-6 cm, papery, reticulately veined, puberulent with white hairs, strigose on veins on both sides, veins 6-8 pairs, curved, base cuneate, attenuate to petiole, margin crenate or sometimes serrate, teeth glandular. Inflorescence axillary in upper 

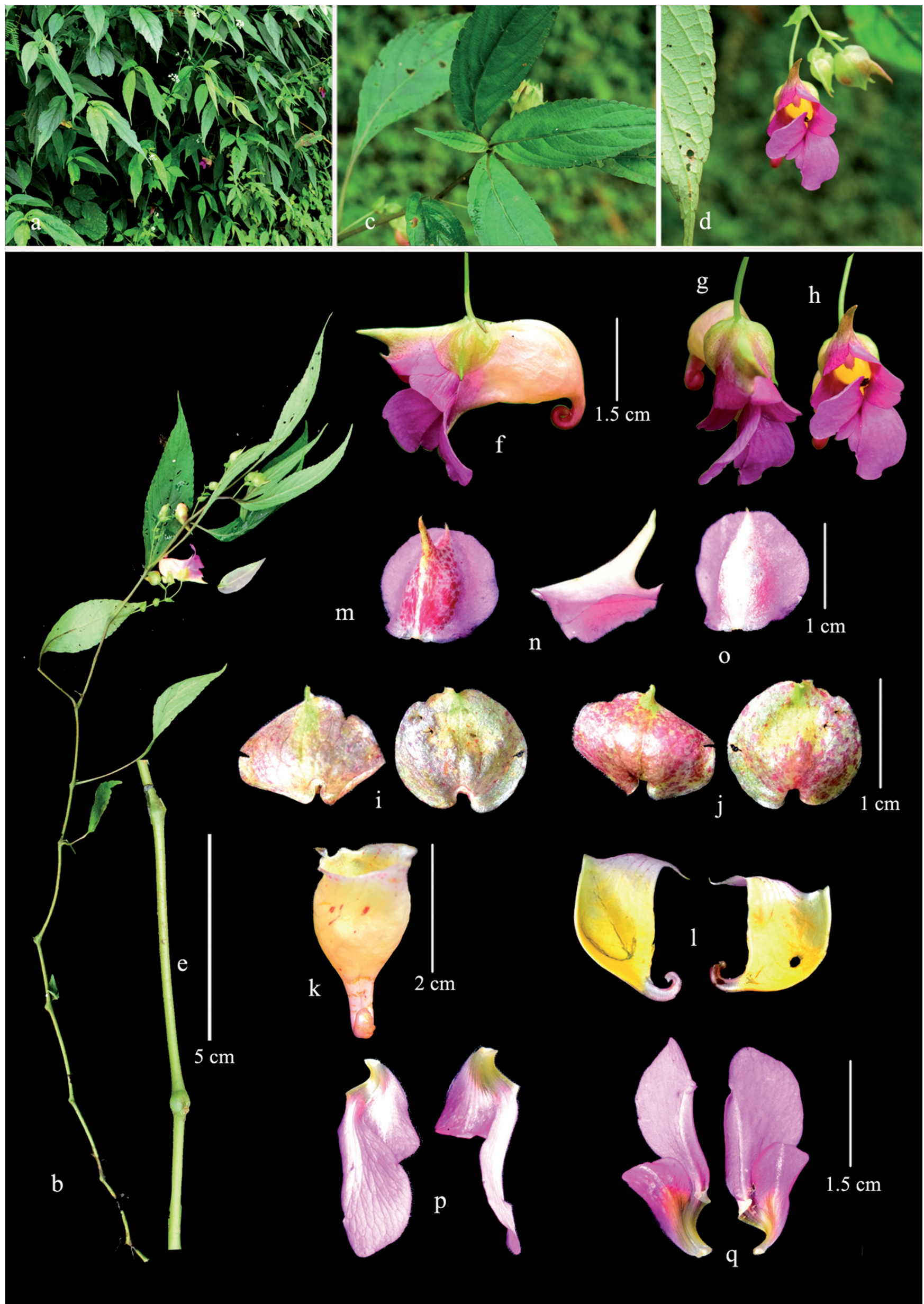

Fig. 1. Impatiens cyclosepala

Explanations: $\mathrm{a}$ - plant in habitat; $\mathrm{b}$ - close up of plant; $\mathrm{c}$ - flower bud; $\mathrm{d}$ - close up of inflorescence; $\mathrm{e}$ - internode; $\mathrm{f}$ - side view of the flower; $\mathrm{g} \& \mathrm{~h}-$ front view of the flower; $\mathrm{I}$ - lateral sepal, ventral side; $\mathrm{j}$ - lateral sepals, dorsal side; $\mathrm{k}$ - lower sepal; 1 - longitudinal section of lower sepal; $\mathrm{m}$ - dorsal petal, top view; $\mathrm{n}$ - dorsal petal, side view with two crista; $\mathrm{o}$ - dorsal petal; $\mathrm{p}$ - lateral united petals dorsal side; $\mathrm{q}$ - lateral united petals ventral side 
part of stem and branches, 5-6 flowered, sometimes branched, peduncles ascending, 6-10 cm long, strigose, equal or shorter than leaves, flower pedicellate, pedicel 1.5-2.5 cm long, strigose, bracteate at base, bract persistent, green, 4-8 mm, thick, 3-4 veined, apex cuspidate, ca. $1 \mathrm{~mm}$, cordate; Flower yellowish pink to purple, $3.5-4 \mathrm{~cm}$ long; lateral sepals 2 , ca. $1 \mathrm{~cm}$ in diameter. Lower sepal broadly saccate, yellowish, ca. $2 \mathrm{~cm}$ deep, abruptly constricted into thick, incurved orange to red spur, 1.2-1.4 cm long in mature flower, mouth oblique, apex ca. $2 \times 1.5 \mathrm{~cm}$, tip acute. Upper petal purple with dark spot, suborbicular, ca. $2 \mathrm{~cm}$ in diameter, abaxial midvein thickened with 2 horn-shaped appendages at middle, outer crista $1 \mathrm{~mm}$ long and inner crista $1 \mathrm{~cm}$ long. Lateral united petals pink, not clawed, ca. 2-2.5 cm, bilobed, basal lobes suborbicular, ca. $1 \mathrm{~cm}$ wide, distal lobes broadly ovate, larger up to $1.5 \mathrm{~cm}$ long, apex obtuse. Filaments linear 4-5 mm, anther acute. Ovary linear, capsule 2-2.5 cm long, linear, 8-10 seeded.

Flowering \& Fruiting: August-October.

$\mathrm{Habitat}$ : It was found growing in moist habitat near a stream in Venia village, Sawa block in East Kameng district and later it was collected from Dirang to Mandala, West Kameng district. Associated species were Impatiens racemosa, Impatiens drapanophora, Strobilanthes attenuata, Quercus sp., Litsea sp., Magnolia sp. etc.

Distribution: India: Arunachal Pradesh, East \& West Kameng district; China.
Conservation Status: Chen et al. (2008) did not give its distribution in Yunnan and also did not mention about the population of species. Based on the herbarium collections, this species was represented by ca. 110 year-old collections and, with this, it has an extended distribution and new species record for India. Based on the distribution records, the authors would like to assess it as an Indeterminate (I) species due to insufficient information on its distribution. However, this species is evaluated here as Endangered [EN, Blab(i, iv)] according to the recent IUCN Red List Criteria and Categories version 3.1 (IUCN, 2012). Further intensive explorations are required in similar habitats in adjacent areas for the possible location of this species. During the present survey, only twelve mature individuals were located, growing as undergrowth in a semi-evergreen forest along a stream. It is possible that I. cyclosepala occurs also in N Myanmar, and other adjoining areas, between original localities in Yunnan and localities described in this paper.

Specimens examined: East Kameng, near Venia village on way to Yakali, Sawa, $1555 \mathrm{~m}$, 28.09.2016, U.K. Tiwari 47777 (ARUN); West Kameng, Dirang, 2402 m, 09.09.2017, K. Chawlu 40908 (ARUN).

Acknowledgements. The authors are grateful to Dr. Paramjit Singh, Director, Botanical Survey of India (BSI), Dr. R. Gogoi, Central National Herbarium (CNH), BSI, Howrah and Head of Office, BSI APRC, Itanagar for facilities and encouragement.

\section{References}

Bhaskar V. 2012. Taxonomic monograph on Impatiens L. (Balsaminaceae) of Western Ghats, South India: the key genus for endemism. 283 pp. Bangalore: Centre for Plant Taxonomic Study.

Borah S., Gogoi R., Satyanarayana P. \& Yu S-X. 2015. Lectotypification of the name Impatiens toppinii Dunn, a new addition of the species for flora of India. Telopea 18: 33-37. https://doi.org/10.7751/telopea8324.

Chen Y. L., Akiyama C. S. \& Ohba H. 2008. Impatiens. In: Z.-Y. Wu, P. H. RAVEn \& D. Y. Hong (eds.). Flora of China, vol. 12, pp. 43-113. Science Press, Beijing \& Missouri Botanical Garden Press, St. Louis, USA.

Dessai J. R. N. \& Janarthanam M. K. 2011. The genus Impatiens (Balsaminaceae) in the northern and parts of central Western Ghats. Rheedea 21: 23-80.
Gogoi R. \& Borah S. 2013a. Impatiens lohitensis, a New Species of Impatiens (Balsaminaceae) from Arunachal Pradesh, India. Taiwania 58: 15-19. DOI: 10.6165/ tai.2013.58.15

Gogor R. \& Borah S. 2013b. Impatiens siculifer Hook. f. (Balsaminaceae) and Tricarpelema chinense D. Y. Hong (Commelinaceae) Two Additions to the Flora of India from Lohit Valley of Arunachal Pradesh, India. Taiwania 58: 146-150. DOI: 10.6165/tai.2013.58.146

Gogor R. \& Borah S. 2014. Impatiens paramjitiana, a new species of Balsaminaceae from Arunachal Pradesh, India. Phytotaxa 175: 171-175. https://doi.org/10.11646/ phytotaxa.175.3.8

Gogor R. \& Borah S. 2015a. Impatiens ashihoi, a new species of Balsaminaceae from Dibang Valley of Arunachal 


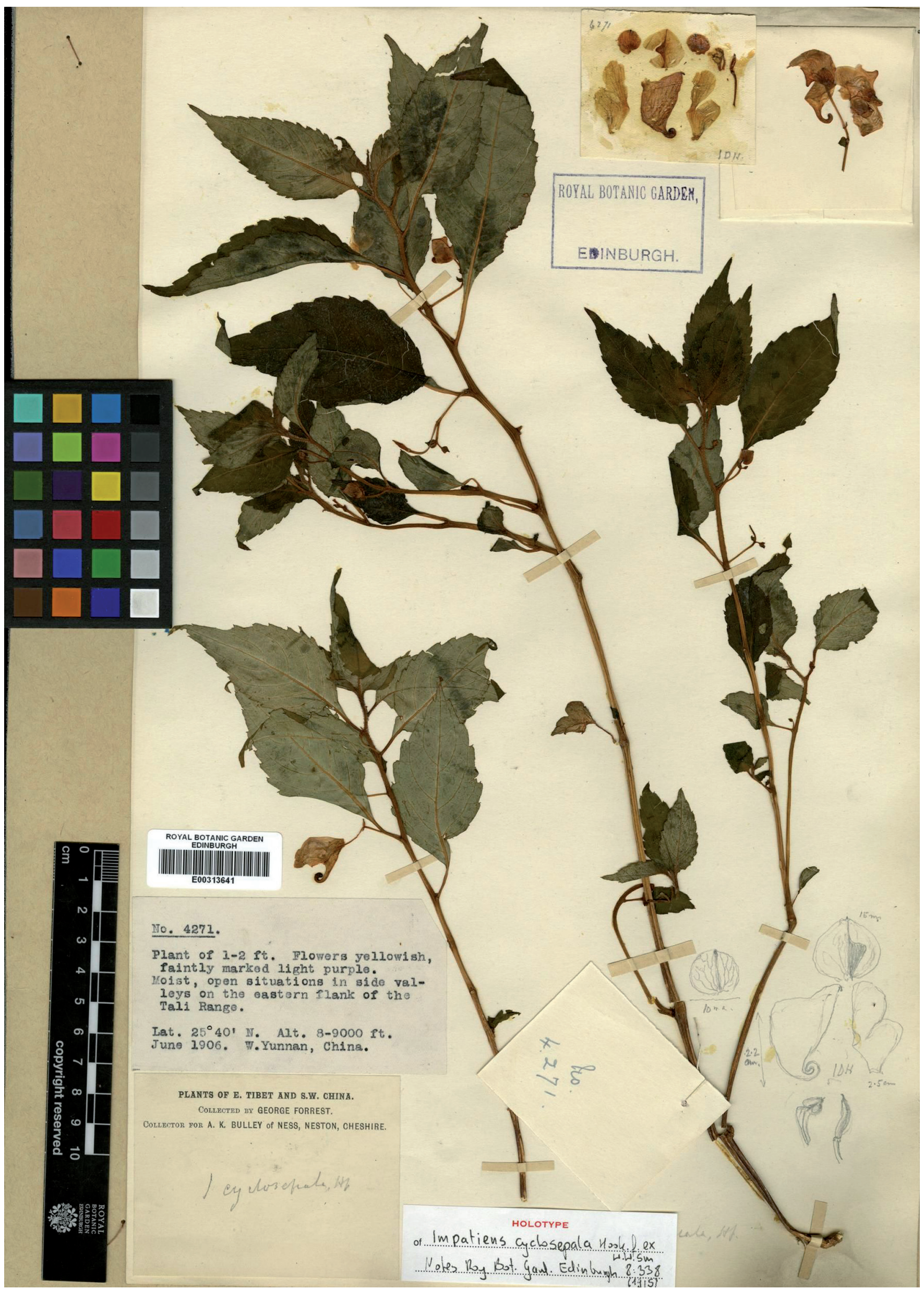

Fig. 2. Impatiens cyclosepala, Holotype: China, West Yunnan, 8-9000 ft, 1906, G. Forrest 4271 E (E00313641), image! 
Pradesh, India. Phytotaxa 238: 278-282. https://doi. org/10.11646/phytotaxa.238.3.7

Gogor R. \& Borah S. 2015b. Impatiens siangensis (Balsaminaceae), a new species from Arunachal Pradesh, India. Phytotaxa 192: 117-120. https://doi.org/10.11646/ phytotaxa.192.2.5

Gogoi R. \& Borah S. 2015c. Impatiens adamowskiana sp. nov.(Balsaminaceae) from Arunachal Pradesh, Northeast India. Nord J Bot 33: 586-590. https://doi. org/10.1111/njb.00798

Gogor R. \& Borah S. 2015d. Impatiens dalaiensis (Balsaminaceae) a new species from Arunachal Pradesh, India. Phytotaxa 207: 286-290. https://doi.org/10.11646/ phytotaxa.207.3.7

Gogor R. \& Borah S. 2015e. Impatiens pathakiana (Balsaminaceae), a new species from Arunachal Pradesh, north-eastern India. Telopea 18: 121-125. https://doi. org/10.7751/telopea8484

Gogor R. \& BoRAH S. 2017a. Impatiens rugosipetala sp. nov. (Balsaminaceae) from Arunachal Pradesh, Northeast India. Phytotaxa 35: 365-367. https://doi.org/10.1111/ phytotaxa.35

Gogor R. \& Borah S. 2017b. Two new species of Impatiens L. (Balsaminaceae) from Arunachal Pradesh, Northeast India. Webbia: Journal of Plant Taxonomy and Geography 72(1): 87-92. http://dx.doi.org/10.1080/0 0837792.2016 .1253913

Gogoi R., Adamowski W., Sherpa N. \& Chhetri G. 2016. On the taxonomic identity and lectotypification of Impatiens uncipetala C.B.Clarke ex Hook.f. Phytotaxa 273(3): 207-211. http://dx.doi.org/10.11646/ phytotaxa.273.3.8

Gogoi R., Barbhuiya H. A. \& Borah S. 2013. Rediscovery of Impatiens laevigata var. grandifolia (Balsaminaceae) from NE India. Taiwania 58: 311-315.

Gogoi R., S. Borah \& Satyanarayana P. 2015a. Impatiens fugongensis, I. yui and I. xanthina (Balsaminaceae), Three New Additions to Flora of India. Journal of Japanese Botany 90: 270-275.

Gogoi R., Hareesh V. S. \& AdAmowski W. 2017a. Impatiens zironiana (Balsaminaceae), a new species from Arunachal Pradesh, northeastern India. Webbia: Journal of Plant Taxonomy and Geography 72(1): 83-86. http://dx.doi.org/10.1080/00837792.2017. 1286773

Gogoi R., Odyuo N. \& Daimary R. 2015b. Impatiens parkinsonii C.E.C. Fisch.(Balsaminaceae), a new addition to flora of India and notes on its typification. Telopea 18: 383-387. http://dx.doi.org/10.7751/telopea9100

Gogoi R., Tham B. B. T., Lidén M. \& Borah S. 2017b. Impatiens pseudolaevigata sp. nov. (Balsaminaceae): a new species from Western Arunachal Pradesh, India. Phytotaxa 313 (2): 227-230. http://dx.doi.org/10.11646/ phytotaxa.313.2.10

Grey-Wilson C. 1989. Impatiens bicornuta and its allies: studies in Balsaminaceae: VII. Kew Bull. 44: 61. http://dx.doi.org/10.2307/4114645

Grey-Wilson C. 1991. Impatiens L. In: A. J. C. Grierson \& D. G. Long (eds.). Flora of Bhutan 2. Royal Botanic Garden, Edinburgh, UK.
Hareesh V. S. \& SABu M. 2017. Impatiens nilalohitae (Balsaminaceae): a new species from northeastern India. Phytotaxa 323(2): 189-193. http://dx.doi. org/10.11646/phytotaxa.323.2.7

Hareesh V. S., Joe A., Gogoi R. \& Sabu M. 2017a. Impatiens arunachalensis (Balsaminaceae), a new species from northeastern India. Phytotaxa 305(1): 047-051. http:// dx.doi.org/10.11646/phytotaxa.305.1.7

Hareesh V. S., Borah S. \& Sabu M. 2017b. Impatiens walongensis (Balsaminaceae) - a new species from North-East India. Nord J Bot 317(3): 226-230. http:// dx.doi.org/10.11646/phytotaxa.317.3.7

Hareesh V. S., Gogoi R. \& Sabu M. 2016a. Impatiens pseudocitrina (Balsaminaceae), a new species from Arunachal Pradesh, Northeast India. Phytotaxa 282: 231-234. http://dx.doi.org/10.11646/phytotaxa.238.3.8

Hareesh V. S., Joe A., Prashob P. \& Sabu M. 2016b. Impatiens roingensis (Balsaminaceae): a new species from northeast India. Webbia: Journal of Plant Taxonomy and Geography 71(2): 187-190. https://doi.org/10.10 80/00837792.2016.1190503

Hooker J. D. 1875. Impatiens L. In: J. D. Hooker (ed.). Flora of British India, vol. 1. L. Reeve \& Co., London, UK.

Hooker J. D. 1908a. Impatiens. In: Hooker's Icones Plantarum, 4th series 9, pp. 1851-1857, Dulau \& co., London.

HoOKer J. D. 1908b. Les espèces du genre "Impatiens" dans l'herbier du Muséum de Paris. Nouv. Arch. Mus. Hist. Nat. Paris, Ser. 4, 10: 232-272, pls. 2-6.

Hooker J. D. 1910. Indian species of Impatiens. Genesis Impatiens species Indicae novae et minus rite cognitae a Cl. A. Meebold detectae. Bull. Misc. Inform. Kew 1910: 291- 300.

Huang S. H., Shui Y. M. \& Chen W. H. 2003. New taxa of Impatiens from Yunnan. Acta Bot. Yunnanica 25: 261-280.

Huang S. H. 2006. Balsaminaceae. In: C. Y. Yu (ed.). Flora Yunnanica, vol. 16, pp. 66-156. Science Press, Beijing.

Lidén M. \& Bharali P. 2017. Impatiens pyrorhiza sp. nov. (Balsaminaceae), a new species from East Himalaya. Nord J Bot 35(4): 411-416. https://doi.org/10.1111/ njb. 01547

Pusalkar P. K. \& Singh D.K. 2010. Three new species of Impatiens (Balsaminaceae) from Western Himalaya, India. Taiwania 55: 13-23.

Ruchisansakun S., Triboun P. \& Jenjittikul T. 2014. A new species of Impatiens (Balsaminaceae) from Southwestern Thailand. Phytotaxa 174: 237-240. http:// dx.doi. org/10.11646/phytotaxa.174.4.5

Swaminathan M. S., Vivekananthan K. \& Nair V. J. 2001. Impatiens. In: N .P. SingH \& D. K. SingH (eds.). Floristic diversity and conservation strategy of India, vol. IV, pp. 2139-2157. Botanical Survey of India, Kolkata.

Toppin S. M. 1920. Notes on the Balsams of Chitral and the Kachin Hills. Bulletin of Miscellaneous Information, Kew 10: 345-367. https://doi.org/10.2307/ 4118598 
VAdAkKoot S. H., Joe A., Gogol R. \& SABU M. 2017. Impatiens arunachalensis (Balsaminaceae), a new species from northeastern India. Phytotaxa 305(1): 047-051. https://doi.org/10.11646/phytotaxa.305.1.7

Vivekananthan K., Rathakrishnan N. C., Swaminathan M. S. \& GHARA L. K. 1997. Balsaminaceae. In: P. K. HAJRA,
R. K. Chakraverty, R. D. Dixit, M. S. Mondal \& U. Chatterjee (eds.). Flora of India, vol. IV, pp. 95-229, Botanical Survey of India, Kolkata.

Yu S. X. 2012. Balsaminaceae of China. Peking University Press, Beijing. 